\title{
Immunotactoid Glomerulopathy
}

National Cancer Institute

\section{Source}

National Cancer Institute. Immunotactoid Glomerulopathy. NCI Thesaurus. Code C158968.

Glomerulonephritis characterized by the presence of Congo-red negative microfibrils in the mesangium and capillary walls of the glomeruli. Morphologic findings include formation of microtubules, evident on electron microscopy, that are larger than those seen in fibrillary g lomerulonephritis (30-50 versus $16-24 \mathrm{~nm}$ in diameter). There may be some overlap in the size of fibrils found in both conditions. 